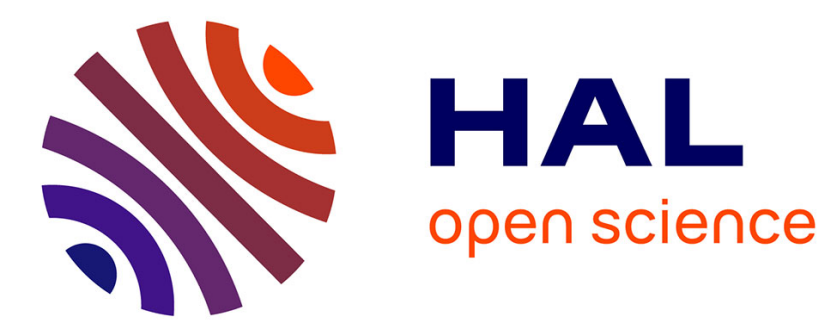

\title{
Minkowski Sum of Ellipsoids and Means of Covariance Matrices
}

Jesus Angulo

\section{To cite this version:}

Jesus Angulo. Minkowski Sum of Ellipsoids and Means of Covariance Matrices. International Conference on Geometric Science of Information GSI 2019, Aug 2019, Toulouse, France. pp.107-115, 10.1007/978-3-030-26980-7_12. hal-02436451

\section{HAL Id: hal-02436451 \\ https://hal.science/hal-02436451}

Submitted on 13 Jan 2020

HAL is a multi-disciplinary open access archive for the deposit and dissemination of scientific research documents, whether they are published or not. The documents may come from teaching and research institutions in France or abroad, or from public or private research centers.
L'archive ouverte pluridisciplinaire HAL, est destinée au dépôt et à la diffusion de documents scientifiques de niveau recherche, publiés ou non, émanant des établissements d'enseignement et de recherche français ou étrangers, des laboratoires publics ou privés. 


\title{
Minkowski Sum of Ellipsoids and Means of Covariance Matrices
}

\author{
Jesús Angulo \\ MINES ParisTech, PSL-Research University, \\ CMM-Centre de Morphologie Mathématique, France \\ jesus. angulo@mines-paristech.fr
}

\begin{abstract}
The Minkowski sum and difference of two ellipsoidal sets are in general not ellipsoidal. However, in many applications, it is required to compute the ellipsoidal set which approximates the Minkowski operations in a certain sense. In this study, an approach based on the so-called ellipsoidal calculus, which provides parameterized families of external and internal ellipsoids that tightly approximate the Minkowski sum and difference of ellipsoids, is considered. Approximations are tight along a direction $l$ in the sense that the support functions on $l$ of the ellipsoids are equal to the support function on $l$ of the sum and difference. External (resp. internal) support function-based approximation can be then selected according to minimal (resp. maximal) measures of volume or trace of the corresponding ellipsoid. The connection between the volume-based approximations to the Minkowski sum and difference of two positive definite matrices and their mean using their Euclidean or Riemannian geometries is developed, which is also related to their Bures-Wasserstein mean.
\end{abstract}

Keywords: minkowski sum; ellipsoid calculus; positive definite matrices

\section{Introduction}

For any pair of sets $X$ and $Y, X, Y \subset \mathbb{R}^{n}$, their Minkowski sum (or addition) $\oplus$ and Minkowski difference (or subtraction) $\ominus$ are defined as follows:

$$
\begin{aligned}
& X \oplus Y=\bigcup_{y \in Y} X_{y}=\{x+y: x \in X, y \in Y\}=\left\{p \in E: X \cap \check{Y}_{p} \neq \emptyset\right\}, \\
& X \ominus Y=\bigcap_{y \in Y} X_{-y}=\left\{p \in E: Y_{p} \subset X\right\}=\left\{x: \forall p \in \check{Y}, x \in X_{p}\right\} .
\end{aligned}
$$

These set operations are fundamental in mathematical morphology [5], since set dilation and erosion of set $X$ by structuring element $B$ are just defined respectively as $\delta_{B}(X)=X \oplus B$ and $\varepsilon_{B}(X)=X \ominus B$. The space of convex sets is closed under Minkowski sum and difference. In this paper, we deal with the particular covex case of ellipsoids and the Minkowski sum and difference of two ellipsoidal sets are in general not ellipsoidal. However, in many applications, we 
are interested in computing the ellipsoidal set which approximates in a certain sense the Minkowski operations between them since the ellipsoids represent a positive (semi-)definite symmetric, a matrix covariance matrix, a Riemannian metric, etc. Indeed, ellipsoidal sets appear nowadays in different imaging techniques, e.g., structure tensor images or DTI. In data analysis, the dispersion of a scatter set of points can be described by a multivariate Gaussian distribution where the covariance matrix may be seen as an ellipsoidal shape centered at the mean position. Ellipsoids are usually taken as canonical sets because they: i) can be concisely described using matrices interpretable as covariance matrices; ii) provide a satisfactory approximation of convex sets in most applications; iii) are invariant under affine transformations.

A classical way to solve the problem will be to, firstly, to compute convex set $S$ corresponding to the Minkowski sum (resp. difference) of two ellipsoids; secondly, to compute the minimum volume ellipsoid that contains $S$, also called the Löwner-John ellipsoid (resp. maximum volume ellipsoid that lies inside a bounded convex set). Both constrained sets are convex semidefinite programming problems which therefore can be solved using classical techniques from convex optimization. Using this approach, little can be said about the set properties of such an approximation to Minkowski sum and difference. In this study, a different approach based on the so-called ellipsoidal calculus [4] is adopted, which is a method for solving problems in control and estimation theory, having unknown but bounded errors in terms of sets of approximating ellipsoidal-value functions. From ellipsoidal calculus (explicit) parameterized families of external and internal ellipsoids that tightly approximate the Minkowski sum and difference of ellipsoids are well formulated. It is also possible to select optimal approximations according to a given criterium. Here we focus in particular on those optimal ellipsoids according to volume.

There are classical results on the topological equivalence between the space of ellipsoids endowed with the Hausdorff metric and the space of their shape matrices endowed with the spectral metric. The goal of this paper is to state another more explicit connection between some particular approximations to the Minkowski sum and difference of ellipsoids and some means between their shape matrices.

\section{Basic Notions on Elipsoidal Space}

Let us assume that everything takes place in the Euclidean space $\mathbb{R}^{n}$. Let $\mathbb{P}(n)$ be the set of positive semidefinite (psd) matrices of size $n \times n$. An ellipsoid, noted by $\mathcal{E}(c, Q)$, in $\mathbb{R}^{n}$, with center $c \in \mathbb{R}^{n}$ and shape matrix $Q \in \mathbb{P}(n)$ is the set

$$
\mathcal{E}(c, Q)=\left\{x \in \mathbb{R}^{n}:\left\langle(x-c), Q^{-1}(x-c)\right\rangle \leq 1\right\} .
$$

Geometrically, an ellipsoid can also be defined as a translated and deformed version of the unit sphere $B_{1}$ of $\mathbb{R}^{n}$, i.e., $\mathcal{E}(c, Q)=c+Q^{1 / 2} B_{1}$. By this parametrization, it is obvious that there is a one-to-one correspondence between ellipsoids and points of the product space $(c, Q) \in \mathbb{R}^{n} \times \mathbb{P}(n)$. 
Hausdorff distance and support function of ellipsoids. The set of subsets of $\mathbb{R}^{n}$ can be metrized by the Hausdorff distance. More precisely, given two nonempty sets $X, Y \subset \mathbb{R}^{n}$, their Hausdorff distance $d_{\mathrm{H}}(X, Y)$ can be defined by means of the Minkowski sum as

$$
d_{\mathrm{H}}(X, Y)=\inf \left\{\lambda \geq 0: X \subseteq Y \oplus B_{\lambda} \text { and } Y \subseteq X \oplus B_{\lambda}\right\}
$$

where $B_{r}$ is the ball of radius $r$ of $\mathbb{R}^{n}$.

The support function $h_{A}$ is a tool for a dual representation of the set as the intersection of half-spaces. The support function $h_{A}: \mathbb{R}^{n} \rightarrow \mathbb{R}$ of a non-empty closed convex set $A \in \mathbb{R}^{n}$ is given by

$$
h_{A}(x)=\sup \{\langle x, a\rangle, a \in A\}, \quad x \in \mathbb{R}^{n},
$$

and it is a real valued, continuous and convex function, satisfiying many relevant properties. In particular, one has:

$$
h_{\alpha A+b}(x)=\alpha h_{A}(x)+\langle x, b\rangle, \quad \alpha \geq 0 ; x, b \in \mathbb{R}^{n} .
$$

The Hausdorff distance $d_{\mathrm{H}}(A, B)$ of two nonempty compact convex sets $A$ and $B$ can be expressed in terms of their support functions:

$$
d_{\mathrm{H}}(A, B)=\sup \left\{\left|h_{A}(x)-h_{B}(x)\right|:\|x\|=1\right\},
$$

which uses the uniform norm on the unit sphere.

For our particular case, the support function of an ellipsoid $\mathcal{E}(c, Q)$ is just given by

$$
h_{\mathcal{E}(c, Q)}(x)=\langle x, c\rangle+\langle x, Q x\rangle^{1 / 2} .
$$

Therefore, given two ellipsoids, $\mathcal{E}\left(c_{1}, Q_{1}\right)$ and $\mathcal{E}\left(c_{2}, Q_{2}\right)$, the Hausdorff distance between them is

$$
\begin{array}{r}
d_{\mathrm{H}}\left(\mathcal{E}\left(c_{1}, Q_{1}\right), \mathcal{E}\left(c_{2}, Q_{2}\right)\right)=\sup _{\|x\|=1}\left\{\left|h_{\mathcal{E}\left(c_{1}, Q_{1}\right)}(x)-h_{\mathcal{E}\left(c_{2}, Q_{2}\right)}(x)\right|\right\} \\
=\sup _{\|x\|=1}\left\{\left|\left\langle x, c_{1}\right\rangle-\left\langle x, c_{2}\right\rangle+\left\langle x, Q_{1} x\right\rangle^{1 / 2}-\left\langle x, Q_{2} x\right\rangle^{1 / 2}\right|\right\} .
\end{array}
$$

It seems clear that for metric purposes, it will be sufficient to study ellipsoids centered at the origin.

Remark on the topology of the space of ellipsoids. Let us review the main result by Goffin and Hoffman [3] on the relationship between the Hausdorff distance and the matrix distance of ellipsoids. Firstly, in order to simplify the notation and avoding the term $1 / 2$ in later expressions, we introduce the following change of variable: $Q \mapsto P=Q^{1 / 2}, P \in \mathbb{P}(n)$. Let $\mathcal{E}\left(c_{1}, P_{1}\right)$ and $\mathcal{E}\left(c_{2}, P_{2}\right)$ be two ellipsoids in $\mathbb{P}^{n}$. Then, define the so-called spectral distance as follows

$$
d_{\text {Spectral }}\left(\mathcal{E}\left(\mathbf{0}, P_{1}\right), \mathcal{E}\left(\mathbf{0}, P_{2}\right)\right)=\left\|P_{1}-P_{2}\right\|_{S},
$$


where $\|\cdot\|_{S}$ is the matrix spectral norm, i.e.,

$$
\|A\|_{S}=\sup \left\{\|A x\|: x \in \mathbb{R}^{n} \text { with }\|x\|=1\right\}=\sqrt{\lambda_{\max }\left(A^{T} A\right)} .
$$

As discussed above on Hausdorff distance, it is sufficient to study ellipsoids centered at the origin. In that case, one has

$$
\begin{aligned}
d_{\text {Spectral }}\left(\mathcal{E}\left(\mathbf{0}, P_{1}\right), \mathcal{E}\left(\mathbf{0}, P_{2}\right)\right) & =\sup _{\|x\|=1}\left\{\left\|\left(P_{1}-P_{2}\right) x\right\|\right\}, \\
d_{\text {Hausdorff }}\left(\mathcal{E}\left(\mathbf{0}, P_{1}\right), \mathcal{E}\left(\mathbf{0}, P_{2}\right)\right) & =\sup _{\|x\|=1}\left\{\left|\left\|P_{1} x\right\|-\left\|P_{2} x\right\|\right|\right\} .
\end{aligned}
$$

Now, the fundamental result is as follows,

Theorem 1 (Goffin and Hoffman, 1983 [3]). Let $\mathcal{E}\left(\mathbf{0}, P_{1}\right)$ and $\mathcal{E}\left(0, P_{2}\right)$ be two centred ellipsoids in $\mathbb{R}^{n}$, with $P_{1}, P_{2} \in \mathbb{P}(n)$. Then

$$
\begin{aligned}
& \quad k_{n}^{-1} d_{\mathrm{S}}\left(\mathcal{E}\left(\mathbf{0}, P_{1}\right), \mathcal{E}\left(\mathbf{0}, P_{2}\right)\right) \leq d_{\mathrm{H}}\left(\mathcal{E}\left(\mathbf{0}, P_{1}\right), \mathcal{E}\left(\mathbf{0}, P_{2}\right)\right) \leq d_{\mathrm{S}}\left(\mathcal{E}\left(\mathbf{0}, P_{1}\right), \mathcal{E}\left(\mathbf{0}, P_{2}\right)\right) \\
& \quad d_{\mathrm{H}}\left(\mathcal{E}\left(\mathbf{0}, P_{1}\right), \mathcal{E}\left(\mathbf{0}, P_{2}\right)\right) \leq d_{\mathrm{S}}\left(\mathcal{E}\left(\mathbf{0}, P_{1}\right), \mathcal{E}\left(\mathbf{0}, P_{2}\right)\right) \leq k_{n} d_{\mathrm{H}}\left(\mathcal{E}\left(\mathbf{0}, P_{1}\right), \mathcal{E}\left(\mathbf{0}, P_{2}\right)\right) \\
& \text { where } k_{n}=2 \sqrt{(2)} n(n+2) .
\end{aligned}
$$

Inequalities from Theorem 1 imply that the two metrics define the same topology on the space of ellipsoids, but, more strongly, the rates of convergence of a sequence of ellipsoids may be studied within a space of sets, or within a space of matrices. In fact, both rates are identical. Additionally, that means that the Hausdorff distance for ellipsoids is essentially a spectral matrix distance.

\section{Ellipsoidal approximations to Minkowski sum and difference of ellipsoids}

Let parameter $l$ be a direction in $\mathbb{R}^{n}, l \in \mathbb{S}^{n-1}$. Given two ellipsoids $\mathcal{E}\left(c_{1}, Q_{1}\right)$ and $\mathcal{E}\left(c_{2}, Q_{2}\right)$, the external and internal ellipsoidal approximation to their Minkowski sum according to direction $l$, noted respectively by $\mathcal{E}\left(c^{\oplus}, Q_{l}^{\oplus,+}\right)$ and $\mathcal{E}\left(c^{\oplus}, Q_{l}^{\oplus,-}\right)$, are tight along the direction $l$ in the sense that the value of support functions at $l$ are equal:

$$
h_{\mathcal{E}\left(c^{\left.\oplus, Q_{l}^{\oplus,-}\right)}\right.}( \pm l)=h_{\mathcal{E}\left(c_{1}, Q_{1}\right) \oplus \mathcal{E}\left(c_{2}, Q_{2}\right)}( \pm l)=h_{\mathcal{E}\left(c^{\oplus}, Q_{l}^{\oplus,+}\right)}( \pm l) .
$$

The center of both approximations is just the vector sum, i.e., $c^{\oplus}=c_{1}+c_{2}$. In the case of the internal ellipsoid, the shape matrix is given by [4]:

$$
Q_{l}^{\oplus,-}=\left(Q_{1}^{1 / 2}+S Q_{2}^{1 / 2}\right)^{T}\left(Q_{1}^{1 / 2}+S Q_{2}^{1 / 2}\right),
$$

with matrix $S$ being orthogonal and vectors $Q_{1}^{1 / 2} l$ and $S Q_{2}^{1 / 2} l$ are parallel. The shape matrix of the external ellipsoid is given by [4]

$$
Q_{l}^{\oplus,+}=\left(1+p^{-1}\right) Q_{1}+(1+p) Q_{2}, \quad p>0,
$$


where $p=\left\langle l, Q_{1} l\right\rangle^{1 / 2} /\left\langle l, Q_{2} l\right\rangle^{1 / 2}$.

Unlike the Minkowski sum, ellipsoidal approximations for the Minkowski difference do not exist for every direction $l$. Similar internal and external approximation for valid directions can be defined in the context of ellipsoidal calculus. See [4] for the expressions.

\subsection{Volume-based optimal approximations}

From these expressions, it is possible to find the direction $l$ such as the corresponding ellipsoids will be optimal according to a given criterion, typically the trace or the volume (i.e., related to the determinant), minimal for the external or maximal for the internal approximations [4]. Let us focus in particular on the approximations of optimal volume. There is a unique ellipsoid of maximal volume contained in the Minkowski sum and its shape matrix is given by [4]

$$
Q_{\max v o l}^{\oplus,-}=Q_{1}+Q_{2}+2 Q_{2}^{1 / 2}\left[Q_{2}^{-1 / 2} Q_{1} Q_{2}^{-1 / 2}\right]^{1 / 2} Q_{2}^{1 / 2} .
$$

Similarly, there is a unique ellipsoid of minimal volume contained in the Minkowski difference and its shape matrix is given by

$$
Q_{\min v o l}^{\ominus,+}=Q_{1}+Q_{2}-2 Q_{2}^{1 / 2}\left[Q_{2}^{-1 / 2} Q_{1} Q_{2}^{-1 / 2}\right]^{1 / 2} Q_{2}^{1 / 2}
$$

\section{Means on Space of $\mathbb{P}(n)$ and Minkowski Sum and Difference}

We discuss in this section an interpretation of the approximations to Minkowski sum and difference in terms of the means of the corresponding shape matrices.

\subsection{Means in two Riemannian Geometries on $\mathbb{P}(n)$}

The standard Riemannian metric distance for any $A, B$ in $\mathbb{P}(n)$ is given by [1]

$$
d_{\text {Riemannian }}(A, B)=\left\|\log \left(A^{-1 / 2} B A^{-1 / 2}\right)\right\|_{2} .
$$

Associated to this distance, the space $\left(\mathbb{P}(n), d s_{\text {Riem }}^{2}\right)$ is a Riemannian manifold where the local metric is the natural metric in the cone:

$$
d s_{\text {Riem }}^{2}=\operatorname{Tr}\left(Q^{-1} d Q Q^{-1} d Q\right) .
$$

Any two points $A, B \in \mathbb{P}(n)$ can be joined by a unique geodesic with respect to this metric:

$$
\gamma_{A, B}^{R i e m}(t)=A^{1 / 2}\left(A^{-1 / 2} B A^{-1 / 2}\right)^{t} A^{1 / 2}, 0 \leq t \leq 1 .
$$


The geometric mean $M_{\text {Riemannian }}(A, B)$ between matrices $A$ and $B$ is evidently the midpoint of this geodesic, i.e.,

$$
M_{\text {Riemannian }}(A, B)=\gamma_{A, B}^{\text {Riem }}(0.5)=A^{1 / 2}\left(A^{-1 / 2} B A^{-1 / 2}\right)^{1 / 2} A^{1 / 2} .
$$

This geometry and the mean for the case of two covariance matrices are well known in information geometry. It corresponds to that of the Fisher metric for the case of Gaussian densities of zero-mean and covariance given by the psd matrix. This mean is symmetric in $A$ and $B$. In fact, it is a kind of symmetrization of the equivalent geometric mean $(a b)^{1 / 2}$ for matrices, since in general $A B \neq B A$. In $\mathbb{P}(n)$, the matrix $A B$ has positive eigenvalues and it has a unique square root $(A B)^{1 / 2}$ that has positive eigenvalues. The eigenvalues of $A B$ are the same as those of $B A$ One has $M_{\text {Riemannian }}(A, B)=A\left(A^{-1} B\right)^{1 / 2}=\left(A B^{-1}\right)^{1 / 2} B$. Thus, one also has [2]

$$
(A B)^{1 / 2}=A M_{\text {Riemannian }}\left(A^{-1}, B\right)=A^{1 / 2}\left(A^{1 / 2} B A^{1 / 2}\right)^{1 / 2} A^{-1 / 2} .
$$

Given $A, B$ in $\mathbb{P}(n)$, the Bures metric distance (in quantum information) and the Wasserstein metric distance (in optimal transport) is

$$
d_{\text {Bures-Wasser }}(A, B)=\left[\operatorname{Tr} A+\operatorname{Tr} B-2 \operatorname{Tr}\left(A^{1 / 2} B A^{1 / 2}\right)^{1 / 2}\right]^{1 / 2} .
$$

If $A$ and $B$ are diagonal matrices (vectors), then $d_{\text {Bures-Wasser }}(A, B)$ reduces to the Hellinger distance between probability distributions. In quantum theory, a density matrix (or state) is a psd matrix $A$ with $\operatorname{Tr} A=1$. Bures distance for density matrices is the particular case of $d_{\text {Bures-Wasser }}(A, B)$. It corresponds to the 2-Wasserstein distance between two Borel probability measures $\mu$ and $\nu$ in $\mathbb{R}^{n}$, when $\mu$ and $\nu$ are zero-mean Gaussian measures with covariance matrices $A$ and $B$.

Bures-Wasserstein distance and the underlying Rieamannian geometry has been recently studied in a deep and illuminating perspective in [2]. The geodesic joining $A$ and $B$ in the Bures-Wassertein metric space is:

$$
\begin{aligned}
\gamma_{A, B}^{B-W}(t) & =(1-t)^{2} A+t^{2} B+t(1-t)\left[(A B)^{1 / 2}+(B A)^{1 / 2}\right] \\
& =A^{-1 / 2}\left[(1-t) A+t\left(A^{1 / 2} B A^{1 / 2}\right)^{1 / 2}\right]^{2} A^{-1 / 2}, 0 \leq t \leq 1 .
\end{aligned}
$$

Therefore, using $t=0.5$ in this geodesic and the equality (6), the BuresWassertein mean of $A$ and $B$ is

$$
\begin{array}{r}
M_{\text {Bures-Wasser }}(A, B)=\frac{1}{4}\left[A+B+(A B)^{1 / 2}+(B A)^{1 / 2}\right]= \\
\frac{1}{2}\left[M_{\text {Euclidean }}(A, B)+\frac{1}{2}\left[A M_{\text {Riemannian }}\left(A^{-1}, B\right)+B M_{\text {Riemannian }}\left(A, B^{-1}\right)\right](7)\right.
\end{array}
$$

where $M_{\text {Euclidean }}(A, B)=\frac{A+B}{2}$ is just the Euclidean (Frobenious norm-based) mean of two matrice in the flat space. 


\subsection{Optimal Approximations to Minkowski Sum and Difference in terms of Means}

Using the notation of the Euclidean and Riemannian means, it it obvious that the internal approximation to the Minkowski sum of maximal volume (3) can be just rewritten as:

$$
Q_{\max v o l}^{\oplus,-}=2\left[M_{\text {Euclidean }}\left(Q_{1}, Q_{2}\right)+M_{\text {Riemannian }}\left(Q_{1}, Q_{2}\right)\right],
$$

and similarly for the external approximation to the Minkowski difference of minimal volume (4):

$$
Q_{\min v o l}^{\ominus,+}=2\left[M_{\text {Euclidean }}\left(Q_{1}, Q_{2}\right)-M_{\text {Riemannian }}\left(Q_{1}, Q_{2}\right)\right] .
$$

Therefore, one has

$$
\begin{aligned}
M_{\text {Euclidean }}\left(Q_{1}, Q_{2}\right) & =\frac{1}{4}\left[Q_{\max v o l}^{\oplus,-}+Q_{\min v o l}^{\ominus,+}\right], \\
M_{\text {Riemannian }}\left(Q_{1}, Q_{2}\right) & =\frac{1}{4}\left[Q_{\max v o l}^{\oplus,-}-Q_{\min v o l}^{\ominus,+}\right] .
\end{aligned}
$$

Euclidean and Riemannian means of covariance matrices are consequently related to the Minkowski sum and difference of the corresponding ellipsoids. This result is not surprising since as we have discussed, the topology of both spaces are equivalent. However, we can observe that the relationship is straightforward in this very particular case.

Furthermore, we can notice that in the case where the matrix product commute, i.e., $A B=B A$, which involves $(A B)^{1 / 2}=M_{\text {Riemannian }}(A, B)$, one just has

$$
Q_{\max v o l}^{\oplus,-}=4 M_{\text {Bures-Wasser }}(A, B) .
$$

A sufficient condition for product commutation is that two matrices are simultaneously diagonalizable. In the case of ellipsoids, it corresponds to the case when they are aligned, i.e., they have the same orientation axis.

\subsection{A Riemannian product space}

For the sake of understanding, let us precise that $Q_{\max v o l}^{\oplus,-}$ does not correspond to the midpoint on a geodesic space product of two copies of $\mathbb{P}(n)$ with the Euclidean and Riemannian metrics. Let us consider the Riemannian manifolds $\left(\mathbb{P}(n), d s_{\text {Euclid }}^{2}\right)$ and $\left(\mathbb{P}(n), d s_{\text {Riem }}^{2}\right)$, where the flat metric is just $d s_{\text {Euclid }}^{2}=d Q^{2}$.

Let us consider now the space $\mathbb{P}(2 n)$, where, on the one hand, for each matrix $Q \in \mathbb{P}(n)$, a map associates it to the matrix $Q^{\times} \in \mathbb{P}(2 n)$ and, on the other hand, the Riemannian metric $g_{\times}=\alpha g_{\text {Euclid }} \otimes \beta g_{\text {Rieman }}, \alpha, \beta>0$, which are respectively given by

$$
Q \mapsto Q^{\times}=\left(\begin{array}{cc}
Q & \mathbf{0}_{n \times n} \\
\mathbf{0}_{n \times n} & Q
\end{array}\right) \quad g_{\times}=\left(\begin{array}{cc}
\alpha g_{\text {Euclid }} & \mathbf{0} \\
\mathbf{0} & \beta g_{\text {Rieman }}
\end{array}\right) .
$$


Note that one has $d s_{\times}^{2}=\alpha d s_{\text {Euclid }}^{2}+\beta d s_{\text {Riem }}^{2}$. Let $A^{\times}$and $B^{\times}$be two different points in this product manifold $\left(\mathbb{P}(2 n), d s_{\times}^{2}\right)$. In this manifold, the Riemannian distance between two points $A^{\times}$and $B^{\times}$is given by

$$
d_{\times}\left(A^{\times}, B^{\times}\right)^{2}=\alpha d_{\text {Euclidean }}(A, B)^{2}+\beta d_{\text {Riemannian }}(A, B)^{2},
$$

where $d_{\text {Euclidean }}(A, B)^{2}=\|A-B\|_{2}^{2}$. In the product manifold, the geodesic from $A^{\times}$and $B^{\times}$is given by

$\gamma_{A, B}^{\times}(t)=\operatorname{diag}\left(\gamma_{A, B}^{\text {Euclid }}\left(\frac{\beta d_{\text {Riemannian }}(A, B)}{d_{\times}(A, B)} t\right), \gamma_{A, B}^{\text {Riem }}\left(\frac{\alpha d_{\text {Euclidean }}(A, B)}{d_{\times}(A, B)} t\right)\right)$,

the scaling of the arc lenght is evident since the lenght of both geodesics is different. In conclusion, the geometry of the space associtated to $Q_{\max v o l}^{\oplus,-}$ as the midpoint of a geodesic is not the trivial product of Euclidean and Riemannian geometry. In any case, since the tangent space of the product manifold $T_{Q \times} \mathbb{P}(2 n)=$ $T_{Q, \text { Euclid }} \mathbb{P}(2) \otimes T_{Q, \text { Rieman }} \mathbb{P}(2)$, the exponential maps of the corresponding spaces can be used to deal with the tangent spaces.

\section{Conclusions and Perspectives}

Ellipsoidal approximations to Minkowski sum and difference are based on the approximation in terms of the support function, which is merely related to approximation in terms of Hausdorff distance. The corresponding metric space of ellipsoids is equivalent to the spectral space of their shape matrices. For a very particular case of optimal approximated ellipsoids in terms of their volume, this equivalence leads to an explicit interpretation based on the mean of the two ellipsoids in two different geometries. Some questions about the underlying Riemannian geometry are still open and deserves additional work. The interest of these approximations to Minkowski sum and difference of ellipsoids in tensorvalued image processing tasks, typically regularization and interpolation, will be explored in ongoing work.

\section{References}

1. R. Bhatia. Positive Definite Matrices. Princeton University Press, 2007.

2. R. Bhatia, T. J., Y. Lim. On the Bures-Wassertein distance between positive definite matrices. Expositiones Mathematicae, To appear 2019.

3. J.-L. Goffin, A. J. Hoffman. On the Relationship between the Hausdorff distance and Matrix distances of Ellipsoids. Linear Algebra and its Applications, Vol. 52-53, 301-313, 1983.

4. A.B. Kurzhanski, I. Vályi. Ellipsoidal Calculus for Estimation and Control. Birkhäuser, Boston, MA, 1996.

5. G. Matheron. Random Sets and Integral Geometry. Wiley, New York, 1975. 